\title{
Ampullary Noninvasive Pancreatobiliary Papillary Neoplasm with Low Grade Dysplasia
}

National Cancer Institute

\section{Source}

National Cancer Institute. Ampullary Noninvasive Pancreatobiliary Papillary Neoplasm

with Low Grade Dysplasia. NCI Thesaurus. Code C95914.

An ampullary noninvasive papillary neoplasm of the pancreatobiliary type characterized by the presence of low grade dysplasia. 